CORRESPONDENCE

Sequential administration of low dose 5-azacytidine (AZA) and donor lymphocyte infusion (DLI) for patients with acute myeloid leukemia (AML) or myelodysplastic syndrome (MDS) in relapse after allogeneic stem cell transplantation (SCT): a prospective study from the Belgian Hematology Society (BHS)

(C) The Author(s), under exclusive licence to Springer Nature Limited 2021

Bone Marrow Transplantation (2022) 57:116-118; https://doi.org/ 10.1038/s41409-021-01464-x

\section{TO THE EDITOR:}

Relapse remains the major cause of failure after allogeneic stem cell transplantation (SCT) and to date, there has been little progress in managing those relapses. Therapeutic options are still limited including supportive care, salvage chemotherapy, donor lymphocyte infusion (DLI), or second SCT [1]. Azacytidine (AZA) has shown to improve survival in patients with MDS and low blast count AML and has been therefore administered in relapsed MDS or AML after SCT [2-4]. Responses appear to be mainly related to an immunomodulatory effect, which consists of upregulated expression of HLA and cancer-associated antigens, and subsequent sensitization of leukemic blasts to an adaptive T-cell response. Moreover, epigenetic mechanisms induce regulatory $\mathrm{T}$ cells, leading to a protective effect against graft-versus-host disease (GvHD) [5-7]. Here are the results of a phase II prospective study conducted on the sequential administration of AZA and DLI in patients with MDS or AML relapsing after SCT.

Adult patients from 18 up to 70 years of age were eligible provided they were transplanted for AML in remission or MDS with less than $10 \%$ marrow blasts, and they developed evidence of relapse with $<30 \%$ marrow blasts. For the first cycle, patients received AZA $100 \mathrm{mg} / \mathrm{m}^{2}$, subcutaneously (SQ) for 5 days. From cycle 2 to $6, A Z A$ was decreased to $35 \mathrm{mg} / \mathrm{m}^{2}$ SQ based on the observations that low dose AZA is sufficient to exert an immunomodulatory effect [7]. DLI was planned on day 1 or 2 of cycles 2, 4, and 6 if free from GvHD to allow lymphocytes to be fully modulated by AZA. The escalating dose schedule of DLI was $5 \times 10^{7}, 1 \times 10^{8}$, and $5 \times 10^{8} \mathrm{CD} 3+/ \mathrm{kg}$ in case of sibling donors, and $5 \times 10^{6}, 1 \times 10^{7}$, and $5 \times 10^{7} \mathrm{CD} 3+/ \mathrm{kg}$ for unrelated donors. In case of complete response (CR) or CR with incomplete blood recovery (CRi), two additional cycles of AZA were administered. If the patient achieved partial response (PR) or stable disease (SD), $A Z A$ was continued until progression (PD) or CR. The primary endpoint was overall response rate (ORR) at 6 months, defined by the achievement of CR, CRi, or PR. Secondary endpoints included overall survival (OS), non-relapse mortality, hematological, and non-hematological toxicities, incidence of GvHD and incidence of infections. Statistical analyses were performed with SPSS 24.0
(SPSS Inc, Chicago, IL, USA) and R 3.4.1 (R Core Team (2017). R: A language and environment for statistical computing. $R$ Foundation for Statistical Computing, Vienna, Austria. URL https://www.Rproject.org/).

A total of 49 analyzed patients has been included in this study. Patients' characteristics are summarized in Table 1. Among them, only 17 (34.7\%) achieved 6 AZA cycles. The main raisons for dropoff were progression $(N=18)$ and infections $(N=3)$. Overall, the median number of administered AZA cycles was 3 (range, 1-12), and 4 patients received additional AZA cycles. The median number of DLI given was 1 (range, $0-3$ ). Twelve out of the 17 patients who completed 6 cycles, received $3 \mathrm{DLI}$.

Overall, 11 patients (22.4\%) showed a response to AZA, including ten $\mathrm{CR} / \mathrm{CRi}$ and one PR. At the time of last follow-up (range, 0-52 months), only three patients were still alive and in remission. At the end of cycle 1, there were five early responders. These patients had a median time to relapse after SCT of 125 days (range, 77-504), and they all relapsed with $<10 \%$ marrow blasts. ORR at day 180 was $29 \%$ [95\% Cl: 27.3-30.6]. In multivariable analysis, only the absence of secondary AML at diagnosis was significantly associated with a better ORR $(\mathrm{HR}=0.39,95 \% \mathrm{Cl}$; $0.19-0.82, p=0.013)$. The median OS was 6 months $[95 \% \mathrm{Cl}$ : 3.2-8.8]. The main causes of death were progression and infection. Median time to progression was 83 days (range, 8-1446). In multivariate analysis, we found that a 9/10 HLA-matched donor $(\mathrm{HR}=2.56$, [95\% Cl: $1.1-6.3], p=0.04)$ and higher bone marrow blasts at relapse $(\mathrm{HR}=2.63$, [95\% Cl: 1.2-5.7], $p=0.01)$ were associated with worse survival, while a longer interval from SCT to relapse correlated with better survival $(\mathrm{HR}=0.41,[95 \% \mathrm{Cl}$ : $0.20-0.83], p=0.013)$. Having received three DLI as a timedependent variable was not associated with survival $(H R=0.93$, [95\% Cl:0.37-2.32], $p=0.87$ ). A total of 38 patients experienced progression during the study period with 27 occurring during AZA. The incidence of acute GvHD was $5.1 \%$ within the entire cohort, and grade II-IV acute GvHD was found in $4.1 \%$. The incidence of chronic GvHD was $12.5 \%$ including three severe. No patient died of chronic GvHD. The cumulative incidence of TRM at day 180 was $12.2 \%$ [95\% Cl: 11.3-13.2]. In multivariate analysis, none of the variables were associated with TRM. Infection was frequent during the study with a median of 1 episode per patient (range, 0-9). Finally, 33 patients (67.3\%) were hospitalized at least once during the study with a median of one hospitalization per patient (range, $0-10$ ).

The median time to relapse after SCT was only 146 days in our cohort, reflecting selection of very aggressive diseases. Median 
Table 1. Patient's characteristics $(N=49)$.

Median follow-up time (range)

Median age (range)

Patients' gender, $N(\%)$

$\mathrm{M} / \mathrm{F}$

Co-morbidities based on HCT-Cl, N (\%)

Performance status at study entrance,

$N(\%)$

ECOG 0

$18(36.7 \%)$

ECOG 1-2

Disease type, $N(\%)$

AML

MDS

Disease status at SCT, $N(\%)$

CR1

CR2

$39(79.6 \%)$

PR

Never treated

$4(8.2 \%)$

$3(6.1 \%)$

$3(6.3 \%)$

Karyotype at diagnosis, $N(\%)$

Intermediate

FLT-3 mutations

NPM1 mutation

Missing

Adverse

Missing

sAML, $N$ (\%)

t-MN, $N(\%)$

Donor type, $N(\%)$

Sibling

Unrelated

HLA matching, $N(\%)$

$10 / 10$

9/10

F donor to $\mathrm{M}$ recipient, $\mathrm{N}(\%)$

Second SCT, $N(\%)$

Conditioning regimen, $N(\%)$

MAC/RIC

Median time from SCT to relapse (range)

Median BM blasts at relapse (range)

Median $\mathrm{Hgb}$ at inclusion (range)

Median WBC at inclusion (range)

Median Plts at inclusion (range)

Median unfractionated donor chimerism at inclusion (range)

Median $\mathrm{CD} 3+$ donor chimerism at inclusion (range)

$N$ number, $M$ male, $F$ female, $A M L$ acute myeloid leukemia, $M D S$ myelodysplastic syndrome, $S C T$ allogeneic stem cell transplantation, $C R 1$ first remission, $C R 2$ second remission, $P R$ partial remission, $S A M L$ secondary AML, $t-M N$ therapy-related myeloid neoplasm, HLA human leukocyte antigen, $M A C$ myeloablative conditioning, $R / C$ reduced-intensity conditioning, $B M$ bone marrow, $\mathrm{Hg} b$ hemoglobin level, WBC white blood cell count, Plts platelets level. $9.9 \mathrm{~g} / \mathrm{dL}(7.1-13.8)$ $2260 / \mu \mathrm{L}(130-34,550)$ $34,000 / \mu \mathrm{L}$ $(4000-447,000)$

$87.6 \%(0-100)$

$89 \%(0-100)$ time to relapse has been reported as the strongest prognostic factor and the reported median survival of such patients is in the range of 6 months [1]. In multivariate analysis, longer median time to relapse and lower marrow blasts at relapse were both significantly associated with better OS, suggesting that more indolent relapses might be better candidates for such therapy $[8,9]$. Even though our selection criteria tried to select upfront better candidates, our ORR $(29 \%)$ is not different from the results of other studies $[2-4,10,11]$ or with standard salvage therapy [1]. In multivariate analysis, only the diagnosis of sAML was associated with a significantly decreased ORR, which is in line with the more resistant profile of such diseases. We observed a few early responders, which may be explained by an immunologic effect from either withdrawal of immunosuppression or upregulation by AZA of several antigens on leukemic cells [7]. Four other responders achieved remission after receiving at least one DLI supporting the potential benefit of additional cell-based therapy $[1,4,9]$. However, in our cohort, there was no significant impact of DLI on neither response nor survival. We think that the concomitant administration of DLI with AZA as in our study might exert a toxic effect on lymphocytes, preventing a full-blown GvL effect but we do not have clinical evidence to prove our hypothesis. Infection was frequent during therapy leading to hospitalization in most cases, but the rate and the type of infection do not appear different from what has been described with AZA given for MDS. We observed a very low incidence of chronic GvHD, which support the protective effect of azacytidine from GvHD [5-7].

Overall, the benefit of our trial remains limited in a very highrisk population. Better outcomes are suggested for patients with more indolent disease and early administration of AZA appears as a promising way to improve efficacy [9]. Moreover, efficacy of AZA in the SCT setting could be improved by the addition of other drugs such as tyrosine kinase inhibitors, venetoclax, or lenalidomide $[8,9]$. We do not have currently enough data to support either pre-emptive or prophylactic strategies, but we believe that patients with a very high risk of relapse could be offered maintenance with hypomethylating agents. However, future trials are needed to identify optimal treatment strategies and to better define high-risk patients.

Xavier Poiré $\mathbb{D}^{1,13 凶}{ }^{凶}$, Carlos Graux ${ }^{2,13}$, Aurélie Ory ${ }^{3}$, Julie Herman ${ }^{3}$, Frédéric Baron (iD ${ }^{4}$, Hélène Schoemans (iD ${ }^{5}$, Philippe Lewalle ${ }^{6}$, Ann De Becker ${ }^{7}$, Dries Deeren $^{8}$, Zwi Berneman ${ }^{9}$, Tessa Kerre ${ }^{10}$ Pierre Zachée ${ }^{11}$, Dominik Selleslag ${ }^{12}$ and Yves Beguin ${ }^{4}$

${ }^{1}$ Section of Hematology, Cliniques Universitaires St-Luc, Université Catholique de Louvain, Brussels, Belgium. ${ }^{2}$ Section of Hematology, CHU UCL Namur-Godinne, Université Catholique de Louvain, Yvoir, Belgium. ${ }^{3}$ Data Management Office, Belgian Hematology Society, Liège, Belgium. ${ }^{4}$ Section of Hematology, Centre Hospitalier Universitaire de Liège, Université de Liège, Liège, Belgium.

Universitaire de Liège, Université de Liège, Liège, Belgium.
${ }^{5}$ Department of Hematology, University Hospitals of Leuven and $\mathrm{KU}$ Leuven, Leuven, Belgium. ${ }^{6}$ Section of Hematology, Institut Jules Bordet, Université libre de Bruxelles, Brussels, Belgium. ${ }^{7}$ Department Clinical Hematology, Universitair Ziekenhuis Brussel, Brussels, Belgium. ${ }^{8}$ Department of Hematology, AZ Delta, Roeselare, Belgium. ${ }^{9}$ Department of Hematology, Antwerp University Hospital, Edegem, Belgium. ${ }^{10}$ Department of Hematology, Ghent University Hospital, Ghent, Belgium. ${ }^{11}$ Department of Hematology, ZNA Stuivenberg, Antwerp, Belgium. ${ }^{12}$ Department of Hematology, AZ Sint Jan, Brugge, Belgium. ${ }^{13}$ These authors contributed equally: Xavier Poiré, Carlos Graux. ${ }^{\boxplus e m a i l: ~ X a v i e r . P o i r e @ u c l o u v a i n . b e ~}$

\section{REFERENCES}

1. Thanarajasingam G, Kim HT, Cutler C, Ho VT, Koreth J, Alyea EP, et al. Outcome and prognostic factors for patients who relapse after allogeneic hematopoietic stem cell transplantation. Biol Blood Marrow Transplant. 2013;19:1713-8. 
2. Craddock C, Jilani N, Siddique S, Yap C, Khan J, Nagra S, et al. Tolerability and clinical activity of post-transplantation azacitidine in patients allografted for acute myeloid leukemia treated on the RICAZA trial. Biol Blood Marrow Transplant. 2016;22:385-90.

3. Jabbour E, Giralt S, Kantarjian H, Garcia-Manero G, Jagasia M, Kebriaei $P$, et al. Low-dose azacitidine after allogeneic stem cell transplantation for acute leukemia. Cancer. 2009;115:1899-905.

4. Schroeder T, Czibere A, Platzbecker U, Bug G, Uharek L, Luft T, et al. Azacitidine and donor lymphocyte infusions as first salvage therapy for relapse of $A M L$ or MDS after allogeneic stem cell transplantation. Leukemia. 2013;27:1229-35.

5. Choi J, Ritchey J, Prior JL, Holt M, Shannon WD, Deych E, et al. In vivo administration of hypomethylating agents mitigate graft-versus-host disease without sacrificing graft-versus-leukemia. Blood. 2010;116:129-39.

6. Cooper ML, Choi J, Karpova D, Vij K, Ritchey J, Schroeder MA, et al. Azacitidine mitigates graft-versus-host disease via differential effects on the proliferation of $T$ effectors and natural regulatory T cells in vivo. J Immunol. 2017;198:3746-54

7. Goodyear OC, Dennis M, Jilani NY, Loke J, Siddique S, Ryan G, et al. Azacitidine augments expansion of regulatory $T$ cells after allogeneic stem cell transplantation in patients with acute myeloid leukemia (AML). Blood. 2012;119:3361-9.

8. Craddock C, Labopin M, Robin M, Finke J, Chevallier P, Yakoub-Agha I, et al. Clinical activity of azacitidine in patients who relapse after allogeneic stem cell transplantation for acute myeloid leukemia. Haematologica. 2016;101:879-83.

9. Schroeder T, Rachlis E, Bug G, Stelljes M, Klein S, Steckel NK, et al. Treatment of acute myeloid leukemia or myelodysplastic syndrome relapse after allogeneic stem cell transplantation with azacitidine and donor lymphocyte infusions-a retrospective multicenter analysis from the German Cooperative Transplant Study Group. Biol Blood Marrow Transplant. 2015;21:653-60.

10. Czibere A, Bruns I, Kröger N, Platzbecker U, Lind J, Zohren F, et al. 5-Azacytidine for the treatment of patients with acute myeloid leukemia or myelodysplastic syndrome who relapse after allo-SCT: a retrospective analysis. Bone Marrow Transplant. 2010;45:872-6.

11. Schroeder T, Rautenberg C, Krüger W, Platzbecker U, Bug G, Steinmann J, et al. Treatment of relapsed AML and MDS after allogeneic stem cell transplantation with decitabine and DLI-a retrospective multicenter analysis on behalf of the German Cooperative Transplant Study Group. Ann Hematol. 2018;97:335-42.

\section{ACKNOWLEDGEMENTS}

Celgene provided azacytidine to all included patients and supported study with a restricted grant. We thank Myriam Labopin, MD, Ph.D., Saint-Antoine, Paris, France who helped us with the statistical analysis.

\section{AUTHOR CONTRIBUTIONS}

$X P$ and $C G$ wrote the paper. $A O$ and $J H$ collected the data. XP analyzed the data and performed the statistical analysis. $X P, C G, F B, H S, P L, A D B, D D, Z B, T K, P Z, D S$, and $Y B$ provided the data. $F B, H S, P L, A D B, D D, Z B, T K, P Z, D S$, and $Y B$ approved the paper.

\section{COMPETING INTERESTS}

FB has received travel grants and/or speaker honoraria from Celgene, AbbVie, Novartis, Pfizer and Sanofi. HS has received travel grants and/or speaker/advisor honoraria from Incyte, Janssen, Novartis, Jazz Pharmaceuticals, Takeda Celgene, AbbVie, MSD, and Therakos. YB has received travel grants and/or speaker/consulting honoraria from Celgene, AbbVie, Novartis, Pfizer, Janssens-Cilag, Amgen and Sanofi. The study was supported by a grant from Celgene Inc.

\section{ADDITIONAL INFORMATION}

Correspondence and requests for materials should be addressed to Xavier Poiré.

Reprints and permission information is available at http://www.nature.com/ reprints

Publisher's note Springer Nature remains neutral with regard to jurisdictional claims in published maps and institutional affiliations. 